\title{
Um Modelo Macrodinâmico de Abertura Comercial, Utilização da Capacidade Produtiva e Crescimento Econômico
}

\author{
Mário Augusto Bertella \\ Gilberto Tadeu Lima
}

Doutor em Economia pelo IE/Unicamp

Professor do Departamento de Economia da FEA-USP

\begin{abstract}
RESUMO
É desenvolvido um modelo macrodinâmico pós-keynesiano para analisar o efeito de variações quantitativas nas tarifas de importação sobre a utilização da capacidade e a taxa de crescimento da economia. Mostra-se que mesmo quando a redução tarifária promove um aumento na produtividade da mão-de-obra e/ou uma queda no mark-up, seu impacto sobre a utilização da capacidade elou a taxa de crescimento pode vir a ser negativo.
\end{abstract}

PALAVRAS-CHAVE

tarifas de importação, utilização da capacidade, crescimento econômico

ABSTRACT

The paper develops a post-keynesian macrodynamic model to analyze the impact of quantitative changes in import tariffs on capacity utilization and growth. It is shown that even in case a tariff reduction happens to lead to a rise in labor produtivity and/or to a fall in markups, it may well have a negative impact on capacity utilization and/or growth.

KEY WORDS

import tariffs, capacity utilization, economic growth

JEL Classification

F4 


\section{INTRODUÇÃO}

Este artigo desenvolve um modelo macrodinâmico pós-keynesiano de análise do impacto de variações no grau de abertura comercial, sob a forma de variações nas tarifas de importação, sobre a utilização da capacidade produtiva e a taxa de crescimento da economia. Em particular, o modelo focaliza a mediação desse impacto pela variação na produtividade da mão-de-obra e/ ou na concentração de mercado (e correspondente mark-up), ambas suportadas por várias evidências empíricas. Porém, mostra-se que mesmo quando a redução tarifária promove um aumento na produtividade da mão-de-obra e/ou uma queda no mark-up da economia, seu impacto último sobre o nível de atividade produtiva e/ou a taxa de crescimento da economia pode vir a ser negativo. Posto que a dinâmica das tarifas de importação, por sua vez, é influenciada pela própria dinâmica da distribuição funcional, fica então estabelecido um canal adicional de influência recíproca entre a distribuição e o crescimento - vale dizer, a distribuição funcional afeta a demanda efetiva em seu componente exportações líquidas e, portanto, também afeta o grau de utilização e o crescimento da capacidade produtiva por meio de seu impacto intertemporal sobre a estrutura tarifária.

Nesse sentido, o presente modelo segue a tradição macrodinâmica pós-keynesiana de considerar a distribuição funcional da renda como um determinante fundamental do crescimento econômico. Embora essa tradição ainda seja mais conhecida pelos modelos elaborados nas décadas de 1950 e 1960 por N. Kaldor, J. Robinson e L. Pasinetti, uma diferenciação deve ser feita entre esses modelos anteriores e formulações mais recentes desenvolvidas por autores mais ligados à tradição de $\mathrm{M}$. Kalecki e J. Steindl, tais como R. Rowthorn (1982) e A. K. Dutt (1984). Na verdade, existe uma diferença básica entre essas duas gerações de modelos pós-keynesianos. Enquanto os modelos da primeira geração assumem implicitamente que no longo prazo a utilização da capacidade produtiva é plena ou dada em um nível normal, nos modelos mais recentes o grau de utilização é plenamente endógeno. Assim, enquanto na geração anterior existe uma relação de longo prazo necessariamente inversa entre o salário real e as taxas de lucro e de crescimento, nos modelos recentes essa relação é normalmente positiva para economias fechadas. Como a propensão a poupar dos assalariados é inferior à dos capitalis- 
tas, um aumento no salário real, ao transferir renda destes para aqueles, eleva a demanda agregada e, na presença de capacidade ociosa, acelera o crescimento econômico.

Numa economia aberta, porém, outros efeitos devem ser considerados, com o que essa relação positiva entre salário real e crescimento econômico pode mesmo deixar de prevalecer. Dependendo da natureza e da intensidade das relações mantidas com o resto do mundo, o crescimento da economia doméstica pode ser afetado negativamente por uma redistribuição pró-salários, mesmo que sua capacidade produtiva não esteja sendo plenamente utilizada. Em Blecker (1989), por exemplo, um aumento no salário real pode vir a reduzir a competitividade externa da economia e, portanto, piorar o saldo comercial numa proporção superior ao efeito demanda positivo derivado daquela redistribuição. Assim sendo, as firmas locais, ao terem que levar em conta a competição externa quando estabelecem suas margens de lucro, vêem reduzida sua capacidade de repassar aumentos de custo por meio de preços mais altos, um elemento igualmente incorporado ao modelo desenvolvido em Sarantis (1990-91). Essa compressão das margens de lucro por parte da competição externa, por outro lado, pode eventualmente comprometer a capacidade de acumulação das firmas e, portanto, a taxa de crescimento da economia. Esta possibilidade emerge igualmente em Bhaduri \& Marglin (1990), em cujo modelo a influência (positiva) da rentabilidade sobre a taxa de acumulação é medida diretamente pela parcela dos lucros na renda, e não pela taxa de lucro.

Embora o modelo desenvolvido a seguir insira-se nessa literatura pós-keynesiana de macroeconomia aberta, procurando assim definir e analisar as condições sob as quais uma redistribuição de renda em favor dos trabalhadores eleva a utilização da capacidade e/ou o crescimento, ele inova ao incorporar aspectos tais como o conflito distributivo na formação de preços e salários, a influência da proteção tarifária sobre a concentração de mercado e a produtividade do trabalho, além da influência da distribuição funcional sobre o nível de proteção tarifária. No compasso desse arcabouço mais inclusivo, são analisadas não somente as propriedades de estabilidade do equilíbrio de curto prazo desses distintos regimes de utilização e crescimento da capacidade produtiva (wage-led e profit-led), mas também as propriedades de estabilida- 
de do equilíbrio do sistema dinâmico centrado no par distribuição e proteção tarifária.

O restante deste artigo tem o seguinte formato. A seção 1 descreve a estrutura do modelo aqui desenvolvido, enquanto a seção 2 o resolve para um determinado momento do tempo. A seção 3, por sua vez, analisa o comportamento dinâmico do modelo, o que é feito com base na focalização das variáveis de estado participação dos salários na renda e tarifa de importação. A última seção resume as principais conclusões obtidas ao longo do artigo.

\section{ESTRUTURA DO MODELO}

Este modelo contempla uma economia aberta sem atividades governamentais. Ela produz um único bem que pode ser utilizado para consumo, investimento e transações com o resto do mundo. Apenas dois fatores de produção são usados, capital e trabalho, combinados em uma tecnologia de produção de coeficientes fixos:

$$
X=\min \left[K u_{k}, L / a\right]
$$

na qual $X$ corresponde ao nível de produto, $K$ é o estoque de capital, $L$ é o nível de emprego, $u_{k}$ a utilização plena da capacidade e $a$ corresponde à relação trabalho-produto.

A produção é realizada por firmas oligopolistas. Elas produzem - e contratam mão-de-obra - de acordo com a demanda, sendo considerado somente o caso em que esta não é suficiente para gerar a plena utilização da capacidade produtiva instalada. Portanto, o nível de emprego é dado por:

$$
L=a X
$$

O investimento desejado das firmas, por sua vez, é representado formalmente por:

$$
g^{i}=\alpha_{0}+\alpha_{1} u+\alpha_{2} \pi
$$


em que $\alpha_{i}$ são parâmetros positivos do investimento desejado, $g^{i}$, expresso como função de $u=X / K$, o grau de utilização da capacidade, e $\pi$, a participação dos lucros na renda. Assume-se que a acumulação desejada depende positivamente do grau de utilização da capacidade devido a efeitos do tipo acelerador, como em Rowthorn (1982) e Dutt (1984, 1990). Como as firmas buscam preservar uma certa margem de capacidade ociosa, elas elevarão seus planos de acumulação de capital sempre que o nível efetivo de ociosidade for menor que o desejado. Porém, enquanto Rowthorn e Dutt seguem Kalecki (1971) e Robinson (1962) e fazem a acumulação desejada depender da taxa de lucro, seguimos Bhaduri e Marglin (1990) ao assumir sua dependência em relação à parcela dos lucros na renda. Segundo eles, isso permite a separação dos efeitos da utilização da capacidade e da parcela dos lucros sobre o investimento, posto que, como veremos em seguida (equação (8)), a taxa de lucro pode ser decomposta entre elas.

As transações comerciais das firmas com o exterior são descritas pelas seguintes funções exportação e importação, respectivamente, ambas normalizadas pelo estoque de capital:

$$
\begin{aligned}
& E=\beta_{0}+\beta_{1} \varepsilon-\beta_{2} u+\beta_{3} u_{f} \\
& M=m_{0}-m_{1} \varepsilon(1+t)+m_{2} u
\end{aligned}
$$

em que $\beta_{i}$ e $m_{i}>0$. O primeiro determinante das exportações é a competitividade ou termos de troca, $\varepsilon$, definido como $e P_{f} / P$, na qual $e$ corresponde à taxa de câmbio nominal, $P_{f}$ é o preço do bem importado em moeda estrangeira e $P$ é o preço do bem doméstico similar em moeda local. Seu sinal é positivo, indicando que aumentos no câmbio real geram aumentos no volume exportado. O segundo fator é o grau de utilização doméstico. Seu sinal é negativo mostrando que uma pressão excessiva da demanda doméstica torna a produção para o mercado local mais rentável e, portanto, deixa capacidade insuficiente para suprir o mercado externo, como argumenta Thirlwall (1986). Por último, temos o grau de utilização do resto do mundo $\left(u_{f}\right)$, e seu sinal é positivo, indicando que aumentos em $u_{f}$ acarretam aumentos nas exportações. Quanto à função importação, seu primeiro determinante é o termo de troca efetivo $\varepsilon^{t}=\varepsilon(1+t)$, já que o produto im- 
portado entra no país taxado por uma alíquota de importação $0 \leq t \leq 1$. Seu sinal é negativo, mostrando que aumentos nesta variável reduzem o volume de bens importados. O segundo e último determinante é o grau de utilização doméstico, e seu sinal é positivo, indicando que aumentos em $u$ acarretam aumentos nas importações. Vale dizer, um aumento (redução) na alíquota de importação $(t)$ representa uma des(valorização) real do termo de troca efetivo $\left(\varepsilon^{t}\right)$ :

$$
\frac{\partial \varepsilon^{t}}{\partial t}=\varepsilon-\varepsilon \frac{(1+z) b e P_{f}(1+t)}{P}>0
$$

em que $(1+z) b e P_{f}(1+t) / P<1$. Assim, a abertura comercial (redução em t) significa uma valorização real do termo de troca efetivo.

Duas são as classes sociais que habitam a economia: capitalistas e trabalhadores. Seguindo a tradição de Marx, Kalecki (1971), Kaldor (1956), Robinson (1962) e Pasinetti (1962), assumem-se distintos comportamentos de poupança e consumo por parte delas. Os trabalhadores ofertam trabalho e ganham apenas salários que são gastos integralmente. Os capitalistas, por sua vez, recebem lucros que são poupados de forma integral. Portanto, a divisão da renda é dada por:

$$
X=\left(\frac{w}{P}\right) L+r K+M e(1+t) \frac{P_{f}}{P}
$$

na qual $w$ é o salário nominal e $M$ o volume de bens importados intermediários, o que significa que a renda é dividida entre capitalistas, trabalhadores e o resto do mundo.

Combinando (2) e (6), a participação dos trabalhadores na renda, $\sigma$, é dada por:

$$
\sigma=V a=1-\pi-b \varepsilon(1+t)
$$

em que $V=w / P$ corresponde ao salário real e $b=M / X$ corresponde à relação bem importado intermediário por unidade de produto. A participação dos trabalhadores na renda também pode ser expressa da seguinte forma: 


$$
\sigma=V a=\frac{1-\delta}{1+z}
$$

na qual $z$ representa o mark-up e $\delta=b e(1+t) P_{f} / w a+b e(1+t) P_{f}$ representa a parcela dos produtos importados no custo unitário total. Assim, a taxa de lucro pode ser expressa por:

$$
r=[1-\sigma-b \varepsilon(1+t)] u=\pi u
$$

em que $\pi=1-\sigma-b \varepsilon(1+t)$ é a parcela dos lucros na renda. Com alguma manipulação algébrica, $\pi$ pode ser alternativamente representado por:

$$
\pi=1-\frac{\sigma}{1-\delta}
$$

ou seja, quanto maior a participação dos produtos importados no custo total, menor a participação dos lucros na renda, dado $\sigma$.

O preço é determinado conforme Kalecki (1971), sendo definido pelas firmas por um mark-up, z, em cima dos custos diretos:

$$
P=(1+z)\left[w a+b e(1+t) P_{f}\right]
$$

O preço está dado em um certo momento do tempo, mas, ao longo do tempo, ele aumentará sempre que a participação efetiva dos trabalhadores na renda for maior que a participação potencial dos trabalhadores na renda resultante do mark-up desejado das firmas $\left(\sigma_{f}\right)_{:}^{1}$

$$
\hat{P}=\theta\left(\sigma-\sigma_{f}\right)
$$

onde $\sigma_{f}=\varphi_{0}-\varphi_{1} t, \operatorname{com} \varphi_{0}, \varphi_{1}>0$, enquanto $0<\theta \leq 1$ é a velocidade de ajustamento e $\hat{P}=\dot{P} / P$. Neste sentido, quanto maior (menor) a tarifa de importação, maior (menor) o mark-up desejado das firmas, menor (maior) a

1 Como pode ser observado pela expressão ( $\left.7^{\prime}\right)$, existe uma relação necessariamente inversa entre $z$ e $\sigma$, para dado $\delta$, de forma que uma maneira de escrever $\hat{P}=\theta\left(z_{f}-z\right)$ é por meio da expressão (10), na qual $z_{f}$ corresponde ao mark-up desejado das firmas. 
participação potencial dos trabalhadores na renda resultante do mark-up desejado das firmas e, portanto, maior a taxa de variação proporcional dos preços.

Num dado momento do tempo, o salário nominal está dado, e como a mãode-obra está sempre em excesso de oferta, o nível de emprego é determinado pela demanda de trabalho. Ao longo do tempo, porém, o salário nominal crescerá sempre que a parcela de renda desejada pelos trabalhadores $\left(\sigma_{w}\right)$ superar a parcela efetiva:

$$
\hat{w}=\psi\left(\sigma_{w}-\sigma\right)
$$

em que $\sigma_{w}=\gamma_{0}+\gamma_{1} u$ e $\gamma_{0}, \gamma_{1}>0,0<\psi \leq 1$ é a velocidade de ajustamento dos salários e $\hat{w}=\dot{w} / w$. Ou seja, a participação desejada pelos trabalhadores depende positivamente da utilização da capacidade.

Num dado momento do tempo, a produtividade do trabalho está dada. A evolução da produtividade, porém, depende da tarifa de importação:

$$
h=-\hat{a}=h_{0}-h_{1} t
$$

Ao indicar uma relação negativa entre a taxa de crescimento da produtividade e a tarifa de importação, buscamos incorporar a noção de que uma redução (aumento) nas alíquotas de importação provocará uma elevação (redução) na produtividade do trabalho ao longo do tempo. Ou seja, a abertura comercial faz com que os produtos produzidos localmente tornem-se mais competitivos para enfrentar seus congêneres estrangeiros. Outro argumento é que uma menor tarifa de importação promove a entrada de bens de capital de nível tecnológico mais elevado, o que eleva o crescimento da produtividade. $^{2}$

2 Evidências empíricas para esses impactos da abertura econômica sobre o mark-up e/ou a produtividade do trabalho podem ser encontradas em HAY (1997), EDWARDS (1998), ROSSI \& FERREIRA (1999), MOREIRA (1999) e KRUEGER (2000).

Est. econ., São Paulo, 34(2): 297-320, abr-jun 2004 
Num dado momento do tempo, o nível das tarifas está dado. Ao longo do tempo, porém, esse nível variará sempre que a distribuição de renda efetiva diferir da distribuição de renda desejada pelo governo, $\sigma_{g}$ :

$$
\hat{t}=\lambda\left(\sigma-\sigma_{g}\right)
$$

em que $\lambda>0{ }^{3}$

Esta formalização procura incorporar a idéia de que um mark-up efetivo superior ao desejado pelo governo levará este último a reduzir a tarifa de importação, de modo a conter qualquer elevação nos mark-ups (e, portanto, qualquer elevação na participação dos lucros na renda) por ele considerada exagerada ou perversa, sob o ponto de vista distributivo.

\section{COMPORTAMENTO ESTÁTICO DO MODELO}

No curto prazo, um conjunto de variáveis é tomado como dado. Assim, o estoque de capital, $K$, a oferta de trabalho, $N$, a relação trabalho-produto, $a$, o nível de preços, $P$, o salário nominal, $w$, a taxa de câmbio nominal, $e$, a relação bem importado intermediário-produto, $b$, a tarifa de importação, $t$, e o preço em moeda estrangeira do bem importado, $P_{f}$, são considerados dados.

A existência de capacidade ociosa implica que o grau de utilização se ajusta para eliminar qualquer excesso de demanda ou oferta na economia. No equilíbrio de curto prazo, portanto, prevalece:

$$
g^{i}=g^{s}-(E-M)
$$

Substituindo as equaçóes (3), (4) e (5) na (14), e lembrando que $r=\pi u$ e $\pi=1-V a-b \varepsilon(1+t)$, ou seja, a participação dos lucros na renda, $\pi$, é complementar à participação dos trabalhadores somada à participação dos produtos importados na renda, temos:

3 Uma formulação análoga a esta seria escrever $\hat{t}=\lambda\left(z_{g}-z\right)$, na qual $z_{g}$ corresponde ao mark$u p$ desejado pelo governo e $z$ ao mark-up efetivo. 
$u^{*}=\frac{\left(\alpha_{0}+\beta_{0}-m_{0}\right)+\alpha_{2}[1-\sigma-b \varepsilon(1+t)]+\left[\beta_{1}+m_{1}(1+t)\right] \varepsilon+\beta_{3} u_{f}}{[1-\sigma-b \varepsilon(1+\mathrm{t})]+\beta_{2}+m_{2}-\alpha_{1}}$

No que concerne à estabilidade do equilíbrio de curto prazo, empregamos o mecanismo de ajustamento keynesiano, no qual o produto mudará como resposta ao excesso de demanda no mercado de bens, o que significa que o valor de equilíbrio de curto prazo será estável, desde que o denominador da expressão acima seja positivo (na vizinhança do ponto de equilíbrio, a sensibilidade dos vazamentos de demanda em relação ao grau de utilização é maior que a sensibilidade das injeções de demanda em relação ao grau de utilização). Adicionalmente, assumimos que o valor correspondente ao saldo comercial, obtido pela substituição de $u^{*}$ nas expressões (4) e (5), e realizando a subtração entre ambas, caso seja negativo, não o será numa magnitude que não possa ser compensado, quer por reservas acumuladas, quer por influxos de capitais correntes. Quanto ao câmbio nominal, no equilíbrio estacionário, assume-se que a sua variação é suficiente para garantir uma relativa constância dos termos de troca efetivos.

Os exercícios de estática comparativa interessantes referem-se ao grau de utilização do resto do mundo $\left(u_{f}\right)$, à participação dos trabalhadores na renda $(\sigma)$, ao câmbio real $(e)$ e ao nivel das tarifas de importação $(t) .{ }^{4}$ A derivada parcial em relação a $u_{f}$ é positiva, indicando que aumentos no grau de utilização do resto do mundo aumentam o grau de utilização doméstico via aumento das exportações. O sinal da derivada parcial do grau de utilização doméstico em relação à participação dos trabalhadores na renda é ambíguo:

$$
\frac{\partial u^{*}}{\partial \sigma}=\frac{u^{*}-\alpha_{2}}{D}
$$

em que $D$ corresponde ao denominador da expressão de $u^{*}$. Assim, a condição para a economia estar num regime wage-led ou estagnacionista $\left(u_{\sigma}^{*}>0\right)$ será satisfeita se $u^{*}>\alpha_{2}$, ou seja, se o grau de utilização de equilíbrio for superior à sensibilidade do investimento em relação à parcela de lu-

4 Os cálculos correspondentes indicaram que não existem restrições cruzadas que reduzam, ainda que parcialmente, as ambigüidades nos sinais das derivadas parciais das expressões que se seguem. 
cros. Caso contrário, a economia se encontrará num regime denominado profit-led $\left(u_{\sigma}^{*}<0\right)$. Quando a parcela dos trabalhadores na renda aumenta, $\mathrm{o}$ consumo agregado se eleva. Porém, quando $\sigma$ aumenta, a participação dos capitalistas na renda cai, dada a participação dos produtos importados na renda. Se o grau de utilização doméstica for maior (menor) que a sensibilidade do investimento em relação à $\pi\left(\alpha_{2}\right)$, então o investimento aumentará (cairá), o que elevará (diminuirá) $u$, resultando no regime wage-led (profitled).

Em relação ao câmbio real, o sinal da derivada parcial de $u^{*}$ é também ambíguo:

$$
\frac{\partial u^{*}}{\partial \varepsilon}=\frac{\beta_{1}+m_{1}(1+t)+b(1+t)\left(u^{*}-\alpha_{2}\right)}{D}
$$

Observe-se que se o regime for wage-led, $u_{\varepsilon}^{*}>0$. Ou seja, uma desvalorização do câmbio real acelera o grau de utilização. Embora um aumento no câmbio real reduza a participação dos lucros na renda, dado $\sigma$, ele também pode contribuir para acelerar o grau de utilização por meio do efeito positivo sobre as exportações líquidas. Sendo o regime wage-led, o grau de utilização é maior que a sensibilidade do investimento em relação à $\pi$, resultando num aumento do investimento.

Por outro lado, se o regime for comandado pelos lucros, a elevação no câmbio real poderá ou não redundar num aumento do grau de utilização. Da mesma forma que uma desvalorização do câmbio real pode contribuir para aumentar as exportações líquidas e, portanto, acelerar o grau de utilização, ela também reduz a participação dos lucros na renda, para dado $\sigma$, o que afeta negativamente o investimento. Daí o resultado líquido dessas duas forças opostas ser ambíguo sobre o grau de utilização doméstico.

Em relação às tarifas, o sinal a derivada parcial de $u^{*}$ é também ambíguo:

$$
\frac{\partial u^{*}}{\partial t}=\frac{\varepsilon\left[m_{1}+b\left(u^{*}-\alpha_{2}\right)\right]}{D}
$$


Da mesma forma que acima, quando o regime for wage-led, uma variação em $t$ produz uma variação no grau de utilização no mesmo sentido. Apesar de uma redução nas tarifas aumentar a participação dos lucros na renda, dado $\sigma$, ela também pode influenciar, de maneira desfavorável, as exportações líquidas, via aumento das importações. Como o regime é wage-led, o grau de utilização é maior que $\alpha_{2}$, causando uma queda no investimento agregado e, portanto, uma queda em $u^{*}$. Se o regime for comandado pelos lucros, o resultado final sobre o grau de utilização é ambíguo, pois embora uma queda em $t$ aumente a participação dos lucros na renda, para dado $\sigma$, ela também pode elevar as importações, o que contribui para reduzir o grau de utilização doméstico e, portanto, o volume de investimento. Daí a indefinição em termos de sinal dessa derivada parcial.

Para obtermos a taxa de crescimento de equilíbrio, $g^{*}$, basta substituirmos as expressões (4), (5), (8) e (15) na (14):

$$
g^{*}=\left(m_{0}-\beta_{0}\right)+\left[1-\sigma-b \varepsilon(1+t)+\beta_{2}+m_{2}\right] u^{*}-\left[\beta_{1}+m_{1}(1+\mathrm{t})\right] \varepsilon-\beta_{3} u_{f}
$$

A taxa de crescimento doméstica responde positivamente a aumentos no grau de utilização do resto do mundo. Por outro lado, a ambigüidade de sinais ocorrerá quando se alterar a variável distributiva $\sigma$.

$$
\frac{\partial g^{*}}{\partial \sigma}=-u^{*}+\left[1-\sigma-b \varepsilon(1+t)+\beta_{2}+m_{2}\right] u_{\sigma}^{*}
$$

A ambigüidade de sinais estará presente se o regime de utilização for wageled, mas sob o regime de utilização profit-led, um aumento na parcela dos trabalhadores gerará, necessariamente, uma queda no crescimento. Assim, sob o regime profit-led, uma política redistributiva pró-salários provocará uma queda em $g$, embora este resultado será ambíguo sob uma economia comandada pelos salários. Em outras palavras, sob o regime wage-led, quando $\sigma$ aumenta, a utilização da capacidade também se eleva. Entretanto, o impacto sobre a taxa de crescimento $g$ é ambíguo, pois embora $u$ aumente, a participação dos capitalistas na renda cai, o que afeta negativamente o investimento. Assim, o resultado líquido irá depender da magnitude dos valores e da elevação de $u$ contra a redução de $\pi$. Por outro lado, sob o regime profit- 
led, quando $\sigma$ aumenta, o grau de utilização e $\pi$ diminuem, o que reforça a queda na acumulação de capital e na taxa de crescimento econômico.

Em relação ao câmbio real $(\varepsilon)$, a taxa de crescimento da economia também apresenta ambigüidade de sinais:

$$
\frac{\partial g^{*}}{\partial \varepsilon}=-b(1+t) u+\left[1-\sigma-b \varepsilon(1+t)+\beta_{2}+m_{2}\right] u_{\varepsilon}^{*}-\left[\beta_{1}+m_{1}(1+t)\right]
$$

Sob o regime de utilização comandado pelos salários, como $u_{\mathcal{E}}^{*}>0$, o resultado certamente será ambíguo. Um aumento no câmbio real reduz a participação dos lucros na renda, dado $\sigma$, embora eleve $u^{*}$. O resultado final sobre o crescimento dependerá do produto resultante dessas forças contrárias. Sob o regime profit-led, $u_{\varepsilon}^{*}$ poderá ser positivo ou negativo. Se negativo, o impacto de uma variação do câmbio real sobre a taxa de crescimento se dará em sentido oposto. Assim, uma desvalorização do câmbio real provocará uma redução na taxa de crescimento, seja pela redução no grau de utilização, seja pela queda na participação dos lucros na renda. Se $u_{\varepsilon}^{*}>0$, a análise é análoga àquela descrita para o regime wage-led e seu resultado sobre a taxa de crescimento é ambíguo.

No que se refere às tarifas $(t)$, o crescimento também mostra ambigüidade:

$$
\frac{\partial g^{*}}{\partial \mathrm{t}}=-b \varepsilon u^{*}+\left[1-\sigma-b \varepsilon(1+t)+\beta_{2}+m_{2}\right] u_{t}^{*}-m_{1} \varepsilon
$$

Se o regime de utilização for comandado pelos salários, segue-se que $u_{t}^{*}>0$. Portanto, o impacto de uma redução nas tarifas sobre o crescimento poaera ser positivo ou negativo. Quando positivo, isso significa que uma queda nas tarifas reduz o grau de utilização, mas aumenta a participação dos lucros na renda, dado $\sigma$, numa proporção maior que a queda em $u^{*}$, causando um aumento na taxa de crescimento. Se negativo, uma redução nas tarifas diminui o grau de utilização, dado $\sigma$, mas eleva a participação dos lucros na renda. Contudo, o efeito final será negativo sobre a taxa de crescimento, dado que a queda no grau de utilização doméstico mais que compensa o aumento em $\pi$. Se o regime for profit-led, segue-se que $u_{t}^{*}$ poderá ser positivo ou negativo. Se for negativo, uma queda nas tarifas acelera 
o crescimento, dado o aumento em $\pi$. Se $u_{t}^{*}>0$, a análise é igual à descrita acima para o regime wage-led. Assim, neste caso, o resultado sobre a taxa de crescimento é indefinido.

\section{COMPORTAMENTO DINÂMICO DO MODELO}

$\mathrm{Na}$ análise dinâmica assumimos que os valores de equilíbrio de curto prazo das variáveis são sempre obtidos, com a economia movendo-se ao longo do tempo devido à mudanças no nível de preços, $P$, no salário nominal, $w$, na produtividade, $\mathrm{l} / a$, e no nível das tarifas, $t$. Uma forma de acompanhar o comportamento do sistema ao longo do tempo é examinar o comportamento dinâmico das variáveis de estado $\sigma$, a participação dos trabalhadores na renda, e $t$, o nível das tarifas.

O comportamento dinâmico da participação dos salários é dado por:

$$
\hat{\sigma}=\hat{V}-h=\hat{w}-\hat{P}-h
$$

Substituindo as expressões (10), (11) e (12) acima, obtemos:

$$
\hat{\sigma}=-(\theta+\psi) \sigma+\psi \gamma_{1} u+\left(h_{1}-\theta \varphi_{1}\right) t+\left(\psi \gamma_{0}+\theta \varphi_{0}-h_{0}\right)
$$

na qual $u$ é dado pela expressão (15).

Por outro lado, a taxa de variação proporcional das tarifas, com a participação dos trabalhadores desejada pelo governo sendo exógena, é dada pela expressão reproduzida abaixo:

$$
\hat{t}=\lambda\left(\sigma-\sigma_{g}\right)
$$

As equações (13) e (24) constituem um sistema de equações diferenciais lineares em que as variações de $\sigma$ e $t$, ao longo de tempo, dependem dos níveis de $\sigma, t$ e dos parâmetros do sistema. 
A matriz $M$ de derivadas parciais é dada por:

$$
\begin{aligned}
& M_{11}=\frac{\partial \sigma}{\partial \sigma}=-(\theta+\psi)+\psi \gamma_{1} u_{\sigma}^{*} \\
& M_{12}=\frac{\partial \hat{\sigma}}{\partial t}=\psi \gamma_{1} u_{t}^{*}+h_{1}-\theta \varphi_{1} \\
& M_{21}=\frac{\partial \hat{t}}{\partial \sigma}=\lambda \\
& M_{22}=\frac{\partial \hat{t}}{\partial t}=0
\end{aligned}
$$

A expressão (25) é ambígua, pois, dada uma variação no nível de $\sigma$, a taxa de variação proporcional dos preços varia na mesma direção, ao passo que a taxa de variação proporcional do salário nominal poderá variar no mesmo sentido, dado o efeito distributivo sobre o grau de utilização doméstico e, portanto, sobre $\hat{w}$ (vide a expressão (11)). A expressão (26) também é ambígua, pois a variação das tarifas poderá alterar o grau de utilização no mesmo sentido ou em sentido contrário. Por outro lado, a variação das tarifas modifica a taxa de crescimento da produtividade em sentido contrário e a taxa de variação proporcional dos preços no mesmo sentido. Assim, a definição do sinal dessa expressão dependerá do efeito preponderante sobre $\hat{\sigma}$. No caso da expressão (27) e (28), por sua vez, não existe ambigüidade. Vejamos, a seguir, as propriedades de estabilidade do equilíbrio nos regimes wage-led e profit-led.

\section{Regime Wage-led}

Sob esse regime, aumentos da participação dos trabalhadores na renda aceleram o grau de utilização, ou seja, $u_{\sigma}^{*}>0$. Adicionalmente, $u_{t}^{*}$ é positivo. Portanto, os sinais da matriz jacobiana são:

$$
M=\left[\begin{array}{l} 
\pm \pm \\
+0
\end{array}\right]
$$


Para que o determinante e o traço da matriz sejam, respectivamente, positivo e negativo (condições de estabilidade do equilíbrio), é necessário que prevaleça a seguinte combinação de sinais:

$$
M_{11}<0 \text { e } M_{12}<0
$$

isto é, dada uma redução nas tarifas, a taxa de crescimento da parcela dos trabalhadores na renda deve aumentar. Entretanto, um aumento na taxa de crescimento de $\sigma$ resulta em elevação do nível de $\sigma$, e como $M_{11}<0$, então este aumento no nível de $\sigma$ deve provocar uma queda em $\hat{\sigma}$. Em uma análise qualitativa, temos o diagrama de fases ilustrado na Figura 1, cujo equilíbrio é um foco estável. A inclinação da isolinha $\hat{\sigma}=0$, dada por $-\left(M_{11} / M_{12}\right)$, é negativa. Como $\partial \hat{\sigma} / \partial \sigma$ é negativo, um aumento em $\sigma$ implica redução na taxa de crescimento de $\sigma$, o que explica a direção dos vetores horizontais. A isolinha $\hat{t}=0$ é vertical, indicando que $\hat{t}$ não depende de $t$. Como $\partial \hat{t} / \partial \sigma$ é positivo, $\hat{t}$ aumenta conforme $\sigma$ aumenta, o que explica a direção dos vetores verticais. Vale ressaltar que, neste caso, a condição necessária e suficiente para a estabilidade do equilíbrio é que, dada uma redução nas tarifas, a taxa de crescimento da participação dos trabalhadores na renda aumente. Porém, como $M_{11}<0$, sabemos que qualquer elevação no nível de $\sigma$ fará com que a taxa de crescimento da participação dos trabalhadores na renda se reduza. Portanto, a possibilidade de melhoria na distribuição é limitada, levando-se em consideração a estabilidade do equilíbrio. 
FIGURA 1

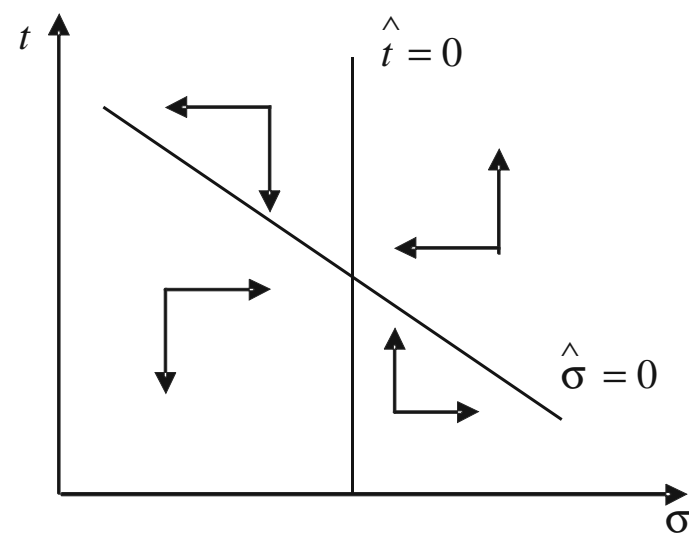

\section{Regime Profit-led}

Sob esse regime, aumentos da participação dos trabalhadores na renda reduzem o grau de utilização doméstico, isto é, $u_{\sigma}^{*}<0$. Os sinais da matriz de derivadas parciais são:

$$
M=\left[\begin{array}{l}
- \pm \\
+0
\end{array}\right]
$$

Para o determinante ser positivo, temos que assumir apenas a hipótese $M_{12}<0$, pois $M_{11}$ já é negativo como decorrência do próprio regime em questão. Nesta situação, o diagrama de fases é qualitativamente semelhante ao anterior.

Comparando-se, portanto, os dois regimes tem-se que as condições de estabilidade são mais rigorosas no regime guiado pelos salários que no profit-led - dados os parâmetros, no regime wage-led são necessárias duas condições para o equilíbrio ser estável, ao passo que no profit-led existe a necessidade de apenas uma condição, qual seja, $M_{12}<0$. Neste sentido específico, podemos concluir que o regime profit-led é mais propenso à estabilidade que o regime guiado pelos salários. 
Até aqui assumimos que a distribuição desejada pelo governo, $\sigma_{g}$, era exógena. Agora assumiremos que $\sigma_{g}$ corresponde à participação desejada pelos trabalhadores na renda - ou seja, $\sigma_{g}=\sigma_{w}$. Assim, a substituição correspondente na expressão (13) gera:

$$
\hat{t}=\lambda \sigma-\lambda \gamma_{1} u-\lambda \gamma_{0}
$$

As equações (24) e (29) constituem um sistema de equações diferenciais lineares no qual as variações de $\sigma$ e $t$, ao longo do tempo, dependem dos níveis de $\sigma, t$ e dos parâmetros do sistema.

A matriz $M$ de derivadas parciais é dada por:

$$
\begin{aligned}
& M_{11}=\frac{\partial \hat{\sigma}}{\partial \sigma}=-(\theta+\psi)+\psi \gamma_{1} u_{\sigma}^{*} \\
& M_{12}=\frac{\partial \hat{\sigma}}{\partial t}=\psi \gamma_{1} u_{t}^{*}+h_{1}-\theta \varphi_{1} \\
& M_{21}=\frac{\partial \hat{t}}{\partial \sigma}=\lambda-\lambda \gamma_{1} u_{\sigma}^{*} \\
& M_{22}=\frac{\partial \hat{t}}{\partial t}=-\lambda \gamma_{1} u_{t}^{*}
\end{aligned}
$$

As expressões (30) e (31) são análogas às expressões (25) e (26), a diferença residindo nas expressões (32) e (33). A expressão (32) é ambígua, pois, dada uma variação no nível de $\sigma$, a taxa de crescimento das tarifas poderá se modificar no mesmo sentido ou não. Por exemplo, um aumento em $\delta$ eleva a taxa de crescimento das tarifas, mas, simultaneamente, poderá aumentar ou reduzir o grau de utilização doméstico, a depender do regime prevalecente (wageou profit-led). A expressão (33) também é ambígua, posto que depende do tipo de regime vigente. No wage-led, $u_{t}^{*}>0$, de forma que $M_{22}<0$, ou seja, um aumento nas tarifas acelera o grau de utilização doméstico, elevando a parcela desejada dos trabalhadores na renda, $\sigma_{w}$, e, portanto, $\sigma_{g}$, já que 
ambos são iguais. Dado $\sigma$, isso provocará uma redução na taxa de crescimento de $\sigma$. No profit-led, como $u_{t}^{*}$ pode ser positivo ou negativo, $M_{22}$ poderá apresentar qualquer sinal. Dada essa enorme ambigüidade, vejamos as situações em que a estabilidade do equilíbrio necessariamente ocorre, a partir da distinção entre os regimes de acumulação.

\section{Regime Wage-led}

Sob esse regime, aumentos da participação dos trabalhadores na renda aumentam o grau de utilização, isto é, $u_{\sigma}^{*}>0$. Além disso, $u_{t}^{*}>0$. Portanto, os sinais da matriz jacobiana são:

$$
M=\left[\begin{array}{l} 
\pm \pm \\
\pm-
\end{array}\right]
$$

A única situação que gera necessariamente a estabilidade do equilíbrio é aquela que considera $M_{11}$ e $M_{12}$ negativos e $M_{21}$ positivo. ${ }^{5}$ Como resultado, essa matriz apresenta os seguintes sinais:

$$
M=\left[\begin{array}{l}
-- \\
+-
\end{array}\right]
$$

ou seja, dada uma redução nas tarifas, a taxa de crescimento da parcela dos trabalhadores na renda deve aumentar. Entretanto, uma elevação na taxa de crescimento de $\sigma$ resulta em aumento do nível de $\sigma$ e, dado que $M_{11}<0$, então esse aumento no nível de $\sigma$ deve provocar uma queda em $\sigma$. Em um diagrama de fases, temos a situação representada na Figura 2.

5 Para os demais sinais da matriz jacobiana, o equilíbrio poderia ser estável, mas não necessariamente. Seria uma possibilidade nas situações em que o $\operatorname{Det}(M)$ e/ou $\operatorname{Tr}(M)$ fosse $(\mathrm{m})$ ambíguo(s). 
FIGURA 2

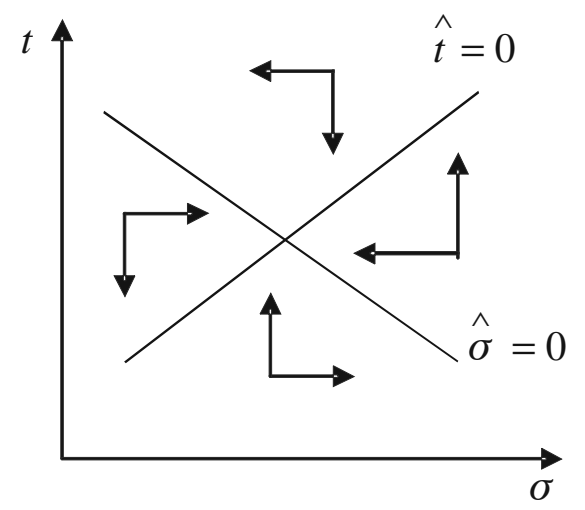

A inclinação da isolinha $\hat{\sigma}=0$, dada por $-\left(M_{11} / M_{12}\right)$, é negativa. Como $\partial \hat{\sigma} / \partial \sigma$ é negativa, $\hat{\sigma}$ cai conforme $\sigma$ se eleva, o que exnlica a direção dos vetores horizontais. A inclinação da isolinha $\hat{t}=0$, dada por $-\left(M_{21} / M_{22}\right)$, é positiva. Como $\partial \hat{t} / \partial \sigma$ é negativa, $\hat{t}$ se eleva quando $t$ se reduz, o que explica a direção dos vetores verticais.

\section{Regime Profit-led}

Sob esse regime, aumentos da participação dos trabalhadores na renda reduzem o grau de utilização. Além disso, $u_{t}^{*}$ pode ser positivo ou negativo. Portanto, os sinais da matriz jacobiana são:

$$
M=\left[\begin{array}{l}
- \pm \\
+ \pm
\end{array}\right]
$$

O único caso em que o equilíbrio é necessariamente estável ocorre quando $M_{12}<0$ e $M_{22}<0 .{ }^{6}$ Desta forma, essa matriz revela os seguintes sinais:

$$
M=\left[\begin{array}{l}
-- \\
+-
\end{array}\right]
$$

6 Idem nota anterior. 
Podemos observar que essa matriz possui os mesmos sinais que o regime guiado pelos salários e que, portanto, o equilíbrio é um foco estável. Portanto, o diagrama de fases correspondente é qualitativamente semelhante ao anterior.

Em relação ao regime wage-led, podemos afirmar que o regime guiado pelos lucros está mais propenso à estabilidade no sentido de que o regime wageled apresenta uma maior quantidade de matrizes (cujos sinais do determinante e/ou traço são inconclusivos) que o regime profit-led. Adicionalmente, podemos observar também que no regime wage-led ou estagnacionista um grande poder de barganha por parte dos trabalhadores, isto é, $M_{11}>0$, poderá ser perverso para a estabilidade do equilíbrio, dado que, nesta situação, o $\operatorname{Det}(M)$ e o $\operatorname{Tr}(M)$ são indefinidos. De outro lado, no regime guiado pelos lucros, $M_{11}$ será sempre negativo, dado que $u_{\sigma}^{*}$ é negativo.

\section{CONCLUSÃO}

Ao contemplar uma economia aberta ao comércio internacional, o modelo aqui desenvolvido focalizou o impacto de variações no grau de abertura comercial, sob a forma de variações nas tarifas de importação, sobre o nível de utilização da capacidade produtiva e a taxa de crescimento da economia, além de suas propriedades de estabilidade.

Observou-se que se o regime de utilização da capacidade produtiva for guiado pelos salários, uma valorização cambial ou uma maior abertura comercial exercem um efeito desfavorável sobre o nível de atividade econômica. Embora uma queda no câmbio real (ou nas tarifas de importação) aumente a parcela dos lucros na renda, ela também contribui para reduzir a demanda agregada mediante uma redução das exportações líquidas. E como o regime é guiado pelos salários, a sensibilidade do investimento em relação à participação dos lucros é menor que o grau de utilização, o que resulta numa desaceleração do nível de atividade. Em relação ao crescimento econômico, sob o regime guiado pelos salários, variações no câmbio real (ou nas tarifas de importação) geram resultado indefinido. Uma queda (aumento) no câmbio real ou nas tarifas aumenta (reduz) a participação dos lucros na renda, em- 
bora reduza (aumente) o grau de utilização da capacidade produtiva. O resultado final sobre a taxa de crescimento dependerá do produto líquido dessas forças opostas.

Por outro lado, se o regime for guiado pelos lucros, variações no câmbio real ou no nível das tarifas de importação resultam em sinal indefinido quanto ao nível de atividade. Da mesma forma que um aumento da tarifa de importação ou do câmbio real acelera a demanda agregada, também reduz a parcela dos lucros na renda, o que afeta negativamente o investimento, contraindo a demanda agregada. Quanto ao crescimento econômico, se o efeito do câmbio real ou das tarifas for negativo sobre o grau de utilização, uma queda no câmbio real ou uma maior abertura comercial aumenta a taxa de crescimento. Assim, uma queda no câmbio real ou na tarifa de importação acelera o nível de atividade, bem como amplia a parcela dos lucros na renda, o que implica aumento da taxa de crescimento. Se o impacto do câmbio real ou das tarifas for positivo sobre o nível de atividade, o resultado final sobre a taxa de crescimento será indefinido, dado que a participação dos lucros na renda se reduzirá. Assim, deve-se registrar que o impacto de uma maior abertura comercial não se traduz, necessariamente, em aumento do crescimento econômico.

No que concerne ao comportamento dinâmico da economia, analisado com base no sistema formado pelas variáveis de estado participação dos trabalhadores na renda e tarifa de importação, observou-se que o regime de utilização da capacidade guiado pelos lucros tende a ser mais propenso à estabilidade que o regime guiado pelos salários. De fato, nota-se uma maior propensão à instabilidade deste último regime apesar de a proteção tarifária estar a serviço dos interesses dos trabalhadores no conflito distributivo.

\section{BIBLIOGRAFIA}

BHADURI, A.; MARGLIN, S. Unemployment and the real wage: the economic basis for contesting political ideologies. Cambridge Journal of Economics, 14, 1990. 
BLECKER, R. International competition, income distribution, and economic growth. Cambridge Journal of Economics, 13, 1989.

DUTT, A. K. Stagnation, income distribution and monopoly power. Cambrige Journal of Economics, 8, 1984.

. Growth, distribution, and uneven development. Cambridge University Press, 1990.

EDWARDS, S. Openness, productivity and growth: what do we really know? Economic Journal, March 1998.

GROSSMAN, G.; HELPMAN, E. Innovation and growth in the global economy. MIT Press, 1991.

HAY, D. The post 1990 Brazilian trade liberalization and the performance of large manufacturing firms: productivity, market share and profits. IPEA, Texto para Discussão 523, 1997.

KALDOR, N. [1959] Alternative theories of distribution. In STIGLITZ, J. E.; UZAWA, H. (eds.), Readings in the modern theory of economic growth. The MIT Press, 1969.

KALECKI, M. Theory of economic dynamics. London: Allen and Unwin, 1954.

. Selected essays on the dynamics of the capitalist economy. Cambridge: Cambridge University Press, 1971.

KRUEGER, A. O. Why trade liberalization is good for growth. In: DIXON, H. D. (ed.), Controversies in macroeconomics: growth, trade and policy. Blackwell Publishers, 2000.

MOREIRA, M. M. Estrangeiros em uma economia aberta: impactos recentes sobre a produtividade, a concentração e o comércio exterior. In: GIAMBIAGI, F.; MOREIRA, M. M. (orgs.), A economia brasileira nos anos 90. Rio de Janeiro: BNDES, 1999.

PASINETTI, L. The rate of profit and income distribution in relation to the rate of economic growth. Review of Economic Studies, 29, 1962.

ROBINSON, J. Essays in the theory of economic growth. Macmillan, 1962.

ROSSI JÚNIOR, J. L.; FERREIRA, P. C. Evolução da produtividade industrial brasileira e abertura comercial. IPEA, Texto para Discussão $651,1999$.

ROWTHORN, R. Demand, real wages, and economic growth. In: SAWYER, M. C. (ed.), Post-keynesian economics. Edward Elgar, 1982.

SARANTIS, N. Distribution and terms of trade dynamics, inflation, and growth. Journal of Post Keynesian Economics, v. 13, n. 2. Winter 1990-91, 
STEINDL, J. Maturity and stagnation in American capitalism. Basil Blackwell, 1952.

TAYLOR, L. Income distribution, inflation, and growth. The MIT Press, 1991.

(mabertella@aol.com)

(giltadeu@usp.br) Agradeço o suporte do CNPq - Bolsa de Produtividade em Pesquisa. (Recebido em janeiro de 2003. Aceito para publicação em outubro de 2003).

Est. econ., São Paulo, 34(2): 297-320, abr-jun 2004 